\title{
$\iota^{-1}$ \\ Lingue Culture Mediazioni \\ Languages Cultures Mediation \\ $8(2021)$ \\ 1
}

La Grecia degli altri: percorsi letterari, geografici e culturali nella Grecia contemporanea

Foreign People's Greece: Literary, Geographic and Cultural Paths in Contemporary Greece

A cura di / Edited by

Luca Gallarini, Dino Gavinelli, Thomas Maloutas, Mauro Novelli

EDITORIALE

Riflessioni e narrazioni attorno alla Grecia: creazioni artistiche, culturali e geografiche

Luca Gallarini, Dino Gavinelli, Thomas Maloutas e Mauro Novelli

Che ci vado a fare in Grecia?

Giuseppe Zanetto

Atene, 1940-1943: italiani e greci nei Quaderni

di Ghiorgos Theotokàs

Massimiliano Maida

Sagapò e Soldatesse: la Grecia degli invasori

Luca Gallarini

"Trascinando muli e sofferenze": la Grecia lontana

di Mario Rigoni Stern

Sergio Di Benedetto

Oriana e i colonnelli: cultura di massa e dittatura greca nell'Italia degli anni Settanta

Alessandro Terreni

Lingue Culture Mediazioni / Languages Cultures Mediation - 8 (2021) 1

https://www.ledonline.it/LCM-Journal/ - Online ISSN 2421-0293 - Print ISSN 2284-1881 
Immaginare la Grecia oggi, fra stereotipi e contro-narrazioni $\quad 97$ (street art e flânerie urbana)

Gilda Tentorio

Carrefours: Migrants' Support Volunteer Tourism in Lesvos

Giovanna Di Matteo

Da Lagkadikia al Mediterraneo: gli spazi delle migrazioni in Grecia Valerio Raffaele

Education Inequalities and Political Behaviour of the Young in Greece in the 2010s

Thomas Maloutas and Maro Pantelidou Malouta

Walk the Wall Athens: An Experiential Walk in the City

Maria Karagiannopoulou

Terra di civiltà e di barbarie: rappresentazioni cinematografiche della Grecia degli altri, tra autenticità e mistificazione

Sara Giovansana

Autori /Authors 


\title{
Terra di civiltà e di barbarie:
}

rappresentazioni cinematografiche della Grecia degli altri, tra autenticità e mistificazione

\section{Sara Giovansana}

DOI: https://dx.doi.org/10.7358/lcm-2021-001-giov

\begin{abstract}
Talking about Greece nowadays does not only mean becoming aware of the immense cultural, social, and linguistic heritage which characterizes the country, but also accepting the huge change which is affecting the nation. The paper focuses on the international cinematographic account on Greece in order to explore the spectrum of non-Greek visions concerning Greek life, history, culture, and traditions. The work is aimed - through the analysis of both American and Italian movies - at outlining stereotypes and authentic elements of the foreign movie industry's representation of "Greekness", browsing some of its most typical leitmotifs. In this respect, noteworthy examples are: the ancient myth; the brutality of war; the cultural misappropriation; the tourism business. The article deals with these issues in an attempt to define possible future developments and points for reflection.
\end{abstract}

Parole chiave: autenticità; cinema; cultura; Grecia; stereotipo.

Keywords: authenticity; cinema; culture; Greece; stereotype.

\section{Delineare un Racconto cinematografico: un'introduzione ALLA GRECIA DEGLI ALTRI}

L'immaginario collettivo orbitante attorno alla Grecia offre immagini che spaziano dalla remota terra dei grandi miti dell'antichità alle località

Lingue Culture Mediazioni / Languages Cultures Mediation - 8 (2021) 1

https://www.ledonline.it/LCM-Journal/ - Online ISSN 2421-0293 - Print ISSN 2284-1881 
balneari delle isole dall'atmosfera mediterranea, passando per la nazione occupata dalle potenze straniere belligeranti durante la Seconda guerra mondiale. Alla cristallizzazione di tali figurazioni hanno contribuito senza ombra di dubbio anche i principali mezzi di comunicazione di massa. Tra di essi, il cinema ricopre un ruolo di spicco, favorendo la proliferazione di nuovi significati e simboli in materia di "grecità" (intendendo con questa definizione quanto appartiene ad una vasta sfera linguistica, letteraria e, in termini generali, alla civiltà ellenica ${ }^{1}$ ), spesso più tesi a rispondere ai gusti e alle predilezioni dell'audience che a restituire un quadro genuinamente autentico della realtà culturale e storica greca.

Per tale ragione, appare interessante un'analisi del racconto cinematografico concernente la Grecia antica, moderna e contemporanea, al fine di scandagliarne cliché e verificarne aderenze all'effettiva realtà. A tal proposito, risulta oltremodo funzionale e utile una riflessione in tema di identità greca, spesso oggetto di decontestualizzazione e mistificazione tra gli avventori occidentali. Considerata europea per eccellenza grazie al suo glorioso passato ellenico ed orientale per via di una forte impronta ottomana (eredità di un'occupazione durata quasi quattro secoli, dal 1453 al 1821), quella greca è una nazione le cui molteplici sfaccettature ne inficiano il preciso inquadramento, alimentando forme di "romanticizzazione" di presunte alterità culturali e contribuendo indirettamente a una sorta di "esotizzazione" delle pratiche di vita e di consumo tipicamente greche (Tzanelli 2003).

L'industria cinematografica ha maturato nel corso degli anni (dalla prima metà del XX secolo ai giorni nostri) uno spiccato interesse verso la raffigurazione di tale eclettico universo, dando vita a un contesto che risulta tanto credibile e plausibile quanto stereotipato e destoricizzato, che dalla messa in scena di un epico passato idealizzato vira verso i resoconti di epoche ben più recenti. In tal senso, utilizzando quale base di partenza la filmografia internazionale (e, più specificatamente, hollywoodiana) e l'offerta cinematografica nazionale, obiettivo primario del presente contributo sarà restituire uno spaccato variegato di tali narrazioni cinematografiche, esplorandone i temi trattati e sottolineandone le criticità, allo scopo di tratteggiare un dipinto il più fedele e articolato possibile della Grecia così come si presenta attraverso la lente di osservazione e ripresa esterna.

Nello specifico, leitmotiv della trattazione saranno due elementi imprescindibili della storia ellenica e della sua raffigurazione cinemato-

${ }^{1}$ https://www.treccani.it/vocabolario/grecita/.

Lingue Culture Mediazioni / Languages Cultures Mediation - 8 (2021) 1

https://www.ledonline.it/LCM-Journal/ - Online ISSN 2421-0293 - Print ISSN 2284-1881 
grafica, ossia le tracce lasciate da una memorabile civiltà e le numerose barbarie di cui il popolo greco è stato vittima nel corso dei secoli. Più precisamente, il lavoro verterà attorno a quattro punti principali: la rivisitazione in chiave nazionalistica della mitologica greca; le cronache (e le omissioni) della violenza al tempo della Seconda guerra mondiale; l'incontro (o scontro, a seconda delle prospettive) tra differenti pratiche di vita, tradizioni e costumi - con gli inevitabili rischi e le conseguenti derive di appropriazione culturale indebita; il viaggio, di cui la Grecia è meta indiscussa. Senza tralasciare alcune doverose considerazioni riguardo all'accoglienza dei prodotti filmici oggetto di discussione da parte del pubblico (greco e non) e degli studiosi in materia, il presente contributo punta ad offrire un quadro generale il più completo possibile delle rappresentazioni filmiche inerenti alla Grecia "degli altri" attingendo dal panorama cinematografico mainstream, indipendente e d'animazione.

\section{Al teMPo DEGLI EROI: I PEPLUM, TRA MITO E LEGGENDA}

Per iniziare questo viaggio attraverso epoche, suggestioni e stili cinematografici differenti tra loro, si è pensato di partire da un genere che più di altri ha contribuito alla "feticizzazione" del mito greco (o, per meglio dire, di una sua reinterpretazione), imprimendo un'impronta decisiva nel repertorio comune di immagini mentali (e mediatiche) relative alla Grecia: il peplum ${ }^{2}$. Anche noto come sword and sandal ("spada e sandalo", due emblemi della cultura materiale del peplum), questo sottogenere di film storici in costume fece la sua comparsa già con il cinema muto degli anni Dieci e Venti del secolo scorso - che regalò al pubblico anche i primi grandi colossal della storia quali Cabiria di Giovanni Pastrone (1914) - per poi raggiungere l'apice del successo negli anni Cinquanta e Sessanta (Cornelius 2011). Incentrate prevalentemente sulla narrazione delle (improbabili) gesta eroiche di uomini dalla forza sovrumana (oppure, seppur non oggetto di interesse per il presente contributo, di storie ambientate in contesti biblici o al tempo dell'Impero Romano), le produzioni statunitensi ad alto investimento di capitale ispirarono registi, sceneggiatori e produttori italiani, dando vita a epopee italiche a basso costo - quanto meno inizialmente - ma con un folto seguito.

2 Da "peplo", tunica utilizzata come abito femminile dalle donne dell'antica Grecia, facile da riprodurre nei reparti costume dei set cinematografici.

Lingue Culture Mediazioni / Languages Cultures Mediation - 8 (2021) 1

https://www.ledonline.it/LCM-Journal/ - Online ISSN 2421-0293 - Print ISSN 2284-1881 
Il peplum, che godette - come anticipato - di particolare fortuna nel mercato cinematografico italiano, lasciò un segno indelebile sull'idea di estetica mitologica greca, al centro di un processo di enfatizzazione e drammatizzazione senza precedenti: mitiche figure greche quali Achille, Ercole e Ulisse interpretate da culturisti di professione dai corpi scolpiti e depilati e dalla pelle oliata e abbronzata (Rushing 2016); pettinature cotonate all'inverosimile; forme fisiche - tanto maschili quanto femminili - e sceniche dagli impressionanti volumi; povere scenografie rese sfarzose da trucchi ottici e specifici macchinari scenici (Di Chiara 2016). Questa tipologia di cinema storico-mitologico con la sua commistione di artificialità e naturalezza (ibid.) e la gradita partecipazione di dive e star statunitensi come Kirk Douglas seppe conquistare sia le platee popolari sia la borghesia altolocata. Mediante il binomio muscoli e testosterone, il genere ridefinì totalmente l'ideale di mascolinità e, ancor di più, della supposta mascolinità nell'antica Grecia, caratterizzata principalmente dalla (semi-)nudità e dallo sfoggio esteriore di una forza quasi ultraterrena e una purezza d'animo interiore, come mostrano opere quali, solo per citare qualche esempio, Le fatiche di Ercole (1958) e Ercole e la regina di Lidia (1959) di Pietro Francisci.

Come testimoniano titoli quali Il Colosso di Rodi di Sergio Leone (1961) e Arrivano i titani di Duccio Tessari (1962) - che scelsero di ripercorrere la via del mito attraverso l'ironia - oppure Ercole al centro della terra di Mario Bava (1961) - indiscusso maestro dell'borror italiano che non mancò di aggiungere un tocco macabro anche a una pellicola di stampo mitologico - vi furono diversi tentativi di svecchiare e arricchire la filmografia del peplum all'italiana, seppur con scarsi risultati (Della Casa 2004). La progressiva riduzione del budget a disposizione e la ripetizione seriale di sequenze narrative (abbinate al reimpiego costante delle medesime scenografie e degli stessi costumi) influirono negativamente sulla qualità delle produzioni, ormai stroncate dalla critica, determinandone il repentino declino. Ciononostante, questa prolifica pagina di cinema popolare italiano offre, soprattutto nella sua prima parte, alcuni importanti spunti di riflessione sul legame tra la rappresentazione dei forzuti dell'antica Grecia e l'ideologia nazionalistica italiana di matrice fascista (Schenk 2006). È, infatti, possibile individuare diverse analogie tra il movimento politico e i principi e le caratteristiche generali dei peplum, che accomunano pellicole imperniate attorno al mito di Roma e pellicole dalle reminiscenze greche (Dell'Agnese 2009).

Un esempio degno di nota a tal proposito riguarda il portamento spavaldo e l'attitudine populista di Benito Mussolini (che, di certo non

Lingue Culture Mediazioni / Languages Cultures Mediation - 8 (2021) 1

https://www.ledonline.it/LCM-Journal/ - Online ISSN 2421-0293 - Print ISSN 2284-1881 
per pura casualità, veniva spesso inquadrato a petto nudo nei filmati di propaganda del tempo): due tratti distintivi di cui era imbevuta la retorica fascista, entro la quale supremazia della corporalità, grottesco machismo e divisioni di genere sembravano essere un netto rimando a uno degli stilemi più classici dei peplum. Paradossalmente, fu proprio per tale ragione che la produzione attraversò una fase di lenta moria nel corso del Ventennio fascista: la figura di Mussolini rappresentava perfettamente, quanto meno nell'immaginario comune, lo stereotipo di virilità dei paladini della giustizia della filmografia sword and sandal, rendendo superflua la loro rappresentazione su schermo (Di Chiara 2016). Il fascismo, dunque, si autoproclamò quale risposta alle fantasie collettive di gloria e grandezza: le stesse utopie sulle quali, del resto, si fondava la narrativa e l'iconografia dei monumentali peplum (Schenk 2006). Non fu, quindi, una mera coincidenza il rilancio con maggior vigore nell'Italia del dopoguerra della produzione in serie di film narranti le mirabolanti avventure dei nerboruti eroi senza macchia e senza colpa della fittizia antica Grecia, intenti a lottare a mani nude contro la tirannia in nome di un popolo sottomesso o a difesa di giovani donne in pericolo: ardue imprese che strizzavano l'occhio alla Resistenza anti-fascista. In altri termini, il genere peplum necessitava di rinnovare la propria immagine agli occhi del pubblico, liberandosi dall'ingombrante e controversa eredità dello strapotere fascista, rappresentato nelle pellicole degli anni Cinquanta e Sessanta nelle vesti del crudele e usurpatore antagonista-nemico, di cui danno una fedele riproduzione opere quali Gli amori di Ercole di Carlo Ludovico Bragaglia (1960) o Ercole contro i tiranni di Babilonia di Domenico Paolella (1964) (D’Amelio 2011).

In tempi decisamente più recenti, il genere epico ha vissuto una sorta di fortunato revival dai risultati decisamente proficui. Titoli da citare a questo riguardo sono indubbiamente Alexander di Oliver Stone (2004), Troy di Wolfgang Petersen (2004), 300 di Zac Snyder (2007) e il suo sequel 300 - L'alba di un impero (300: Rise of an Empire) di Noam Murro (2014). Gli ottimi risultati ottenuti al box office - Troy primo fra tutti, con più di 497.000.000 di dollari incassati - sono da ricondurre più ai cast stellari coinvolti nelle riprese - tra i quali spiccano Colin Farrell, Eric Bana, Brad Pitt e Gerard Butler nei panni degli antichi (e al contempo moderni) eroi in stile peplum - che alla precisa ricostruzione storica, di cui si evidenzia, al contrario, l'inaccuratezza (sintomo, peraltro, di una scarsa conoscenza generale in materia, come la critica ha più volte ribadito). Anche in questo caso, come per i primi peplum, la visione mainstream della Grecia sembra ridursi all'ostentazione di una grandiosa

Lingue Culture Mediazioni / Languages Cultures Mediation - 8 (2021) 1

https://www.ledonline.it/LCM-Journal/ - Online ISSN 2421-0293 - Print ISSN 2284-1881 
potenza; all'esasperazione del vigore e della velocità; alla messa in mostra delle fattezze corporee; alla sfera della sessualità e perfino a una certa "visceralità" (Rushing 2016). Anche analizzando il panorama delle serie TV adolescenziali quali Xena (1995-2001), Hercules (1995-1999) e Young Hercules (1998-1999) - veri e propri fenomeni mediatici degli anni '90 i riferimenti risultano essere sostanzialmente gli stessi (Amato e Dell'Agnese 2015).

Perfino la major statunitense Walt Disney Company nel 1997 tentò la strada del mito greco con il film d'animazione Hercules (diretto da John Musker e Ron Clements): una peculiare rielaborazione della tradizione peplum di cui riprende le convezioni essenziali sopracitate, seppur in un'ottica fanciullesca chiaramente più adeguata a un pubblico infantile (Pallant 2011). Inoltre, più che di mitologia greca (di cui viene riportata la mera caricatura in chiave ironica), la simbologia del film sembra essere intrisa di cultura pop statunitense. Emblematica, in tal senso, è la scena in cui l'inaspettata fama di Hercules si accompagna a un'ondata di merchandising, quasi come se il giovane eroe si fosse tramutato in una celebrità del jet set statunitense e la rivisitazione del mito greco fosse quasi più una strategia di product placement della Walt Disney (Hassapopoulou 2007). Ciò, tuttavia, non stupisce poi molto se si esaminano i Paesi di produzione delle pellicole citate nel precedente paragrafo, tra cui figurano - primi fra tutti - Paesi Bassi, Germania, Regno Unito, Italia, Francia, Malta e naturalmente, Stati Uniti. Ma non la Grecia.

\section{3. "I GRECI E TUTTI QUELLI CHE VORREBBERO ESSERLO": RIPENSARE LA GRECITÀ, TRA CLICHÉ E VERITÀ}

Uno degli esempi indubbiamente più eclatanti attraverso i quali la cinematografia si è confrontata con la cultura greca è Il mio grosso grasso matrimonio greco (My Big Fat Greek Wedding) di Joel Zwick (2002). Ambientato nella Chicago dei giorni nostri, la commedia hollywoodiana di stampo etnico costituisce la quintessenza della relazione tra "grecità" e "americanità", aprendo interessanti scenari al dialogo interculturale. Scritto dalla stessa attrice che interpreta la protagonista della storia (la sceneggiatrice e regista di origini greche Nia Vardalos), il film narra le vicende di Toula Portokalos, una donna trentenne cresciuta negli Stati Uniti in una famiglia greca di vedute conservatrici dalla quale cerca di affrancarsi affermando la sua indipendenza ed autonomia. Fulcro

Lingue Culture Mediazioni / Languages Cultures Mediation - 8 (2021) 1

https://www.ledonline.it/LCM-Journal/ - Online ISSN 2421-0293 - Print ISSN 2284-1881 
della narrazione è la storia d'amore tra la giovane e Ian (un insegnante di letteratura vegetariano, ben educato e di bell'aspetto che incarna alla perfezione il prototipo alto-borghese W.A.S.P. ${ }^{3}$ ), che scatena le ire del nucleo familiare tradizionalista, il quale sottopone l'ignaro professore a una serie di rocambolesche pratiche culturali prima di permettere ai due innamorati di convolare a nozze.

Mediante gag e schermaglie comiche, stando alla critica, il film serve allo scopo di "folklorizzare" il philotimo ${ }^{4}$ greco in un modo quasi artificiale, che disconosce la reale e contemporanea condizione della maggioranza della comunità greco-americana degli Stati Uniti, costituita da una middle-class urbana estranea alle stramberie e agli eccessi. I Portokalos, al contrario, impersonano alla perfezione l'idea statunitense comune (e superficiale) di famiglia greca (Tzanelli 2004): conservatori e dediti al consumo di pantagrueliche quantità di carne, vivono in una casa dipinta di bianco e blu (i colori della bandiera greca, ben piantata nel giardino) e arredata con colonne e non sembrano affatto intenzionati a integrarsi nella società (Iancu 2017). Risulta, quindi, naturale e logico che per Toula l'unica via di fuga consista nel rinunciare al proprio milieu culturale, cambiando completamente il proprio look per assomigliare - a detta sua - alle ragazze puramente americane che frequentano il college con lei, che hanno la pelle bianca, vestono alla moda e che - quanto meno agli occhi di una ragazza greca oppressa da gravosi retaggi culturali - appartengono a un contesto dove non viene richiesto loro di mettere la testa a posto, sposarsi il prima possibile con un uomo che non amano e procreare per obbedire a modelli culturali vetusti e superati (Tzioumakis and Papadimitriou 2016).

La pellicola low-budget ebbe un successo commerciale strabiliante e inatteso, ma non fu risparmiata da critiche pungenti, che la accusavano di scimmiottare la cultura greca attraverso stereotipi e convenzioni capaci di soddisfare tanto la comunità greco-americana quanto il pubblico adulto medio a cui le commedie sentimentali si rivolgono. Il sistema di credenze e le pratiche di vita sembrano essere stati tramutati in caricature di dubbio gusto, benché sia necessario constatare quanto l'ironia faccia dell'esasperazione dei cliché una carta vincente, rendendola addirittura un'arma di (auto-)difesa per minoranze etniche che intendono

3 White Anglo-Saxon and Protestant.

4 Termine sostanzialmente intraducibile, poiché il suo significato corrisponde a una serie di pratiche, virtù e comportamenti legati a concetti quali il patriottismo e l'onore di essere greci.

Lingue Culture Mediazioni / Languages Cultures Mediation - 8 (2021) 1

https://www.ledonline.it/LCM-Journal/ - Online ISSN 2421-0293 - Print ISSN 2284-1881 
manifestarsi al mercato internazionale (Hassapopoulou 2007). Tuttavia, il rischio che una percezione di questo tipo sia sfruttata quale mezzo di subordinazione e marginalizzazione delle alterità culturali è innegabile, come è innegabile il rischio di una scarsa comprensione da parte degli spettatori meno informati, che verosimilmente legittimano tali rappresentazioni come genuinamente autentiche, plausibili o non lesive delle specificità culturali altrui (ibid.). Il film suscitò, infatti, sentimenti contrastanti negli expat greci residenti negli Stati Uniti, tra coloro i quali si sentivano offesi da quella che consideravano una forma di derisione dei loro valori e chi, per converso, si ritrovava in quei rigidi schemi e in quelle rigorose norme sociali e applaudiva alla volontà di realizzazione personale (fondamentale, è necessario sottolinearlo) della protagonista attraverso la socializzazione, il lavoro e il potenziale creativo (Glezakos 2002).

Opinioni discordanti anche quelle riscontrate a seguito dell'uscita nelle sale cinematografiche de Le mie grosse grasse vacanze greche (My Life in Ruins), diretto da Donald Petrie e scritto da Tom Hanks (2009). Al centro delle vicende è Georgia, docente greco-americana di storia classica che ritorna nella patria dei suoi antenati per lavorare presso l'università di Atene. Dopo solo un anno, alcuni tagli alla spesa per l'istruzione (segnale di una situazione economica greca sempre più instabile) la costringono ad accettare un impiego insoddisfacente come guida per turisti poco interessati a spiegazioni erudite e decisamente più attratti dalle cosiddette 4s: sea, sun, souvlaki ${ }^{5}$, sex. Nel film la Grecia si presenta al pubblico in qualità non solo di meta turistica, bensì anche di terra dal potere miracoloso, in grado di aiutare anime in cerca di felicità e di ricucire insanabili ferite del passato. In altre parole, viene perpetuata l'immagine "orientalista" della Grecia quale luogo di misticismo, indomite passioni e irrazionalità (Anagnostou 2015), che cammina su un sottile filo che corre tra una modernità fatta di motociclette e souvenir made in China e una tradizione millenaria che sa di filosofia e primordiale sacralità. La stessa Georgia ritrova in Grecia - tra danze, canti e scenari mozzafiato - quello che quasi inconsciamente la diaspora le aveva tolto: un'opportunità di vera riconnessione con il proprio io interiore e con ciò che la circonda (paesaggio greco incluso).

Tra i landmark per eccellenza di questa idilliaca visione dell'identità nazionale vanno sicuramente citati: l'Acropoli con il suo trionfale ingresso marcato dai Propilei; il maestoso Partenone; il tempio di Apollo

${ }_{5}^{5}$ Popolare street food greco a base di carne e verdure grigliate su uno spiedo.

Lingue Culture Mediazioni / Languages Cultures Mediation - 8 (2021) 1

https://www.ledonline.it/LCM-Journal/ - Online ISSN 2421-0293 - Print ISSN 2284-1881 
a Delfi; l'antica Olimpia; Mykonos con i suoi suggestivi mulini a vento e le inconfondibili chiese tinteggiate di bianco delle Cicladi. Il film funse, dunque, da motore per la promozione turistica della Grecia in un periodo di forte crisi, che non passò inosservato nemmeno alla Banca Europea, che proprio nel 2009 (anno di uscita della pellicola) registrò una riduzione del 15\% circa del numero dei turisti (Tzanelli and Korstanje 2016). L'eventualità di un ritorno economico allettò perfino lo Stato greco, che per la prima volta concesse a una troupe cinematografica di filmare presso i monumenti storici (Tzioumakis and Papadimitriou 2015). Il risultato finale è una sorta di incontro-scontro tra l'anti-modernismo occidentale in tema di Grecia e il presente della nazione, in una mescolanza culturale entro la quale si palesano affinità e conflitti, familiarità ed estraneità. Pur non mancando di alcuni topoi tipici di un certo affresco ellenico astorico, quali la comunione con la natura e la ritrovata pace dei sensi, l'opera di Petrie è da considerarsi un tentativo nel complesso riuscito di negoziazione tra sfere culturali diverse, sulle quali il film invita a riflettere con l'auspicio di un maggior sincretismo futuro (Basea 2012; Tzioumakis and Papadimitriou 2015).

\section{IN VIAGGIO ALLA RICERCA DI SÉ: LA GRECIA COME META TURISTICA}

Come si può dedurre da quanto scritto nei paragrafi precedenti, quello tra industria cinematografica e industria turistica è un legame intramontabile, al punto che si arriva a parlare di cineturismo, di cui Mamma mia! di Phillyda Lloyd (2008) - capolavoro del genere musical che vanta attori del calibro di Meryl Streep, Julie Walters, Pierce Brosnan e Colin Firth - rappresenta un caso caratteristico. Tra indimenticabili canzoni degli ABBA e momenti di ilarità mista a commozione, il film diede popolarità a livello globale a due piccole isole dell'arcipelago delle Sporadi Skiathos e Skopelos - scelte quali location, modificandone ritmi e stili di vita. I sostanziali cambiamenti innescati a livello socio-economico (come spesso accade parlando di globalizzazione) hanno reso i paradisi naturali di Skiathos e Skopelos due destinazioni sempre più mondane, dove non mancano i locali notturni e - naturalmente - le agenzie turistiche che propongono al turista medio pacchetti all-inclusive con tour guidati presso i luoghi più iconici del musical (Tzanelli 2017). Tra spiagge immacolate, acque cristalline e pittoreschi porticcioli, le isole in questione costituiscono l'apoteosi dell'evasione dal proprio quotidiano che

Lingue Culture Mediazioni / Languages Cultures Mediation - 8 (2021) 1

https://www.ledonline.it/LCM-Journal/ - Online ISSN 2421-0293 - Print ISSN 2284-1881 
il vacanziere - e, più nello specifico, il vacanziere diretto in Grecia - ricerca, qui pienamente appagato da alcuni scorci suggestivi che il film non manca di fissare su schermo: Kastani e Glysteri; la chiesa di Agios Ioannis sto Kastri; la penisola montuosa di Pelion con il grazioso porto di Damouchari e la Torretta della Campana di San Nikolaos. Ritorna, dunque, il tema del viaggio affrontato nelle pagine precedenti, di cui il racconto cinematografico relativo alla Grecia degli altri non manca certo di ragguardevoli esempi.

La Messenia, regione storica del Peloponneso sud-occidentale dominato dal monte Taigeto, è il perfetto sfondo per la storia d'amore tra Jesse e Celine, protagonisti del dramma sentimentale Before Midnight di Richard Linklater (2013). Le emozionanti vedute dalla villa dell'autore di racconti di viaggi Patrick Leigh Fermor (tra i più grandi cultori della materia greca che la storia abbia mai conosciuto) a Kardamyli, la meravigliosa Navarino e lo sperduto villaggio di Platsa con il limitrofo castello di Methoni ${ }^{6}$ formano l'impeccabile contorno al prologo della trilogia di Linklater composta da Prima dell'alba (Before Sunrise) - uscito nelle sale nel 1995 - e Prima del tramonto (Before Sunset) - datato 2004. Persino il cinema italiano non ha disdegnato il connubio fatto di suggestive località turistiche (prevalentemente balneari) greche e viaggi alla ricerca di se stessi, come dimostrano titoli quali Immaturi - Il viaggio di Paolo Genovese (2012) e Che ne sarà di noi di Giovanni Veronesi (2004) - ambientati rispettivamente a Paros e Santorini - che riprendono il classico espediente narrativo della spensierata vacanza post-maturità dalla quale si ritorna con un bagaglio arricchito di nuove emozioni ed esperienze formative.

Con queste scelte cinematografiche, la Grecia conferma la sua decennale tradizione nell'ambito dell'ospitalità che per l'economia greca ha un'indubbia valenza, nonostante alcune limitazioni dovute alla complessa morfologia del territorio, alla carenze di infrastrutture ricettive e alla problematicità dei collegamenti (Kapiki 2012). Uno sguardo attento alle quattro pellicole qui prese a campione al fine di introdurre tale tematica svela un valore quasi terapeutico del viaggio in terra greca, il cui inestimabile patrimonio detiene una sorta di facoltà ristoratrice che infonde energia e speranza alle anime in cerca della propria strada (Basea 2012). Un Paese senza tempo, dunque, quasi uno spazio "eterotopico" (Binetti 2015) - ben definito, eppure incredibilmente differente da qualsiasi altro spazio - che da semplice background si fa personaggio principale delle

${ }^{6}$ https://www.cntraveler.com/galleries/2014-03-02/oscars-greece-before-midnightethan-hawke-julie-delpy.

Lingue Culture Mediazioni / Languages Cultures Mediation - 8 (2021) 1

https://www.ledonline.it/LCM-Journal/ - Online ISSN 2421-0293 - Print ISSN 2284-1881 
storie raccontate con il quale lo spettatore si può identificare. Di fatto, $\mathrm{i}$ flussi turistici rispondono positivamente a queste "spettacolarizzazioni" del paesaggio e delle ricchezze greche che preparano un fertile terreno per interessanti sviluppi futuri (Hudson and Brent Ritchie 2005).

\section{Dolore e Bellezza: LA Grecia Degli Altri Al TEMPO DELLA GUERRA}

Una delle pagine senz'altro più tragiche della storia greca si scrisse durante la Seconda guerra mondiale e più precisamente nel corso dell'occupazione italo-tedesca della Grecia. Correva l'anno 1940 quando Benito Mussolino diede il via alla Campagna italiana di Grecia con un'offensiva dell'esercito italiano di stanza in Albania atta a riaffermare il ruolo italiano nel conflitto bellico e a rinsaldare l'alleanza con il Reich tedesco guidato da Adolf Hitler (Santarelli 2004). La tattica studiata dal dittatore italiano - inficiata dalla pessima situazione logistica e dalla disorganizzazione delle truppe - si risolse in un nulla di fatto e fu respinta dalle forze militari greche. Solo l'intervento nell'aprile del 1941 della Wehrmacht tedesca - passato alla storia come Operazione Marita - scongiurò la capitolazione dell'Italia e risolse la "questione balcanica", segnando l'inizio di una traumatica occupazione della Grecia da parte delle forze dell'Asse, che instaurarono un regime collaborazionista di stampo totalitario (scioltosi solo anni dopo, nel 1944) chiamato Stato Ellenico, capitanato dal generale Georgios Tsolakoglu (Gerolymatos 2016). Triste simbolo dell'invasione, la svastica tedesca che sventolava sull'Acropoli (Stockings and Hancock 2013): la barbarie che ammorbava la civiltà. Esempi cinematografici che propongono diverse letture delle atrocità e dei crimini di guerra compiuti in quel periodo sono: Il mandolino del Capitano Corelli (Captain's Corelli Mandolin) di John Madden (2001); Mediterraneo di Gabriele Salvatores (1991) e Appartamento ad Atene di Ruggero Dipaola (2012).

Quella dipinta nei capolavori di Madden e Salvatores - che, seppur con modalità differenti, si interfacciano con l'assalto (nazi-)fascista in Grecia - è una conturbante e benevola terra "donna" e "madre", che accoglie e perdona anche il nemico offrendogli un mondo ovattato lontano dalle brutture della guerra, dove la musica e il calcio annullano le differenze culturali e linguistiche, nazionali o regionali che siano (Van Watson 2005). L'Italia e la Grecia ritrovano nel dramma l'antico lega-

Lingue Culture Mediazioni / Languages Cultures Mediation - 8 (2021) 1

https://www.ledonline.it/LCM-Journal/ - Online ISSN 2421-0293 - Print ISSN 2284-1881 
me che le accomuna e quella solidarietà che le unisce (esemplificativo in tal senso il detto italiano "una faccia, una razza", citato anche nell'opera di Salvatores): una somiglianza che annulla le distanze e assolve la prima dalle proprie colpe nei confronti della seconda. Un esotismo magico, quello greco, che sortisce l'effetto contrario rispetto a quello previsto: non è l'Italia a conquistare la Grecia, ma è la Grecia a conquistare tanto il distaccamento militare italiano occupante ne Il mandolino del Capitano Corelli quanto gli otto soldati italiani dimenticati dalla madrepatria nel mar Egeo e dediti all'ozio e ai piaceri della carne di Mediterraneo (Van Watson 2005). Ancora una volta, la Grecia è un'ibrida e fluida frontiera di scambio e ritrovo, dove le divisioni etniche e identitarie cessano di esistere in nome di una pacifica coesistenza: un'utopia che sembra destinata a durare in eterno, un transitorio e frugale stato di serenità fuori dal tempo, che viene bruscamente interrotto da una realtà fatta di disillusione e sofferenza (Binetti 2015).

Girate rispettivamente a Cefalonia e a Kastellorizo (ufficialmente Megisti), le pellicole firmate da Madden e Salvatores furono tacciate di eccessivo semplicismo e negazionismo (sponsorizzando il falso e stereotipato ideale degli “italiani brava gente”), benché - è importante sottolinearlo - siano prodotti filmici possibili di svariate interpretazioni, soprattutto nel caso di Mediterraneo, che appare quasi più la sofferta dichiarazione di fallimento di un'intera generazione che aveva cercato di cambiare il mondo senza riuscirvi. Tale convenzione comune d'innocenza, comunque, disconosce le deprecabili azioni compiute dalle truppe italiane ai danni del popolo greco, al quale non viene resa giustizia (Hope 2009; Van Watson 2005). Sarebbero, dunque, i complici tedeschi i soli e unici responsabili, rei di essersi macchiati di colpe di gran lunga peggiori. Appartamento ad Atene di Ruggero Dipaola ne offre una straziante versione, resa ancor più evocativa dall'ambientazione prescelta: il quartiere Plaka di Atene, alle pendici dell'Acropoli (ricostruita) che si intravede in tutto il suo tacito e amaro splendore nel corso del film. Qui, nell'anno 1943, vivono gli Helianos, una famiglia greca un tempo agiata e ora ridotta alla fame, che si vede costretta a dividere la propria abitazione con lo spietato capitano tedesco Kalter, che si insinua nella loro vita conducendoli verso la distruzione. Si tratta, dunque, di pellicole che si approcciano a un tema tanto delicato come la ferita greca provocata dalla violenza con modalità differenti, offrendo visioni distinte e ciononostante ben lontane dall'essere univoche.

Lingue Culture Mediazioni / Languages Cultures Mediation - 8 (2021) 1

https://www.ledonline.it/LCM-Journal/ - Online ISSN 2421-0293 - Print ISSN 2284-1881 


\section{Conclusioni}

Al termine di questo viaggio (una parola rivelatasi fondamentale per la presente trattazione) attraverso la Grecia degli altri, risulta opportuno ricongiungere ipotesi e suggestioni presentate nelle pagine precedenti tendendo un filo invisibile che in qualche modo le colleghi. Alla luce dell'importanza che i mass media ricoprono nel plasmare percezioni collettive e nell'influenzare idee generalmente condivise, le tematiche oggetto d'indagine nel presente elaborato acquisiscono un rilevante significato. A fronte delle molteplici raffigurazioni filmiche raccolte, si può constatare come, in relazione alla Grecia, da qualche anno a questa parte l'industria dell'intrattenimento si sia chiusa in un silenzio imperscrutabile, che non solo dimostra quanto sia limitata la globale rappresentazione visuale dell'altro in termini culturali, ma che apre anche la strada a interessanti quesiti, a cui vale la pena cercare di dare una risposta. A tal proposito, risultano particolarmente utili le teorie presentate da Marina Hassapopoulou, docente di Studi cinematografici presso la New York University, nel contributo "It's all Greek to Me": Misappropriations of Greekness in American Mass Media (2007), il cui titolo è già per natura piuttosto paradigmatico: "It's all Greek to me", infatti, è un'espressione della lingua inglese che sta ad indicare un'impossibilità di comprensione reciproca, che effettivamente sembra essere il problema principale alla base del rapporto tra cinema internazionale e Grecia. Sarebbe, di fatto, da attribuire a questo complessivo fraintendimento la stereotipata concezione della storia e della cultura ellenica che ha indirizzato l'industria cinematografica verso una rappresentazione che è tanto stereotipata quanto scarna - benché sia oggetto di dibattito quale delle due derive sia da considerarsi più negativa o lesiva verso l'autenticità altrui (Hassapopoulou 2007). Eppure, dalla Grecia giungono straordinarie dimostrazioni filmiche della complessità e profondità sociale, culturale e spaziale greca, che si discostano nettamente dalla perpetuazione persistente del ristretto ventaglio di figurazioni convenzionali di cui il presente contributo ha cercato di presentare i leitmotiv più canonici.

Basta osservare attentamente gli sviluppi della new wave cinematografica greca per rendersi conto dell'assoluta ricchezza di tematiche offerte di cui il pubblico internazionale ha una conoscenza decisamente ridotta. Si pensi solo a Kynodontas del celebre e versatile Yorgos Lanthimos (2009): un'opera aberrante e disturbante (foriera della crisi socio-economica che avrebbe devastato da lì a pochi mesi la Grecia) che pone in risalto tematiche attuali per l'Europa intera quali la crescita, lo

Lingue Culture Mediazioni / Languages Cultures Mediation - 8 (2021) 1

https://www.ledonline.it/LCM-Journal/ - Online ISSN 2421-0293 - Print ISSN 2284-1881 
scontro padri-figli, l'impossibile separazione dal nucleo familiare. Una generazione senza punti di riferimento, sfiduciata e oppressa quella di Lanthimos, che si può ritrovare anche in pellicole di sottile ferocia quali Miss Violence di Alexandros Avranas (2013) e Attenberg di Athina Rachel Tsangari (2010). La metafora della crisi economica e identitaria greca ha veicolato il malcontento e la paura verso un'urgenza creativa senza pari, che produce inaspettati ritratti della Grecia contemporanea. Persino un tema tanto inflazionato - come si ha avuto modo di vedere nelle pagine precedenti - quanto quello del turismo viene caricato di inaspettate valenze nella cornice della nuova onda cinematografica greca. Argyris Papadimitropoulos ne è il massimo esempio, giacché spezza definitivamente l'abusato legame tra paradisiache gemme dell'Egeo e spensierate vacanze all'insegna del divertimento e dell'introspezione ambientando su una di queste piccole isole (Antiparos, per la precisione) uno scioccante e macabro thriller dal tocco autoriale intitolato Suntan (2016). Racconti cinematografici tra loro divergenti e per questo singolari, che al di fuori della Grecia non godono ancora della risonanza necessaria per rinnovare uno spettro di prodotti filmici che sembrano ancora troppo vincolati alle mistificazioni passate e che gravano pesantemente su un'innovativa rappresentazione del presente.

\section{RIFERIMENTI BIBLIOGRAFICI}

Amato, Fabio, e Elena Dell'Agnese, a cura di. 2014. Schermi Americani. Geografia e geopolitica degli Stati Uniti nella serie televisive. Milano: Unicopli.

Anagnostou, Yiorgos. 2015. "Within the Nation and Beyond: Mediating Diaspora Belonging in My Life in Ruins". FILMICON: Journal of Greek Film Studies 3: $1-31$.

Basea, Erato. 2012. "My Life in Ruins: Hollywood and Holidays in Greece in Times of Crisis". Interactions Studies in Communication \& Culture 3 (2): 199-208.

Binetti, Vincenzo. 2015. "A Mediterranean Heterotopia: Gabriele Salvatores and the Poetics of Fleeing". Mediterranean Studies 23 (1): 74-85. doi: 10.5325/ mediterraneanstu.23.1.0074.

Cornelius, Michael G., ed. 2011. Of Muscles and Men: Essays on the Sword and Sandal Film. Jefferson: McFarland \& Company.

D’Amelio, Maria Elena. 2011. "Hercules, Politics, and Movies". In Of Muscles and Men: Essays on the Sword and Sandal Film, edited by Michael G. Cornelius, 15-27. Jefferson: McFarland \& Company.

Lingue Culture Mediazioni / Languages Cultures Mediation - 8 (2021) 1

https://www.ledonline.it/LCM-Journal/ - Online ISSN 2421-0293 - Print ISSN 2284-1881 
Della Casa, Stefano. 2004. "Peplum”. Enciclopedia del cinema. [10/04/2021]. https:// www.treccani.it/enciclopedia/peplum_\%28Enciclopedia-del-Cinema\%29/.

Dell'Agnese, Elena. 2009. Paesaggi ed eroi. Cinema, nazione e geopolitica. Torino: UTET University Press.

Di Chiara, Francesco. 2016. Peplum. Il cinema italiano alle prese col mondo antico. Roma: Donzelli.

Gerolymatos, André. 2016. An International Civil War: Greece, 1943-1949. New Haven - London: Yale University Press.

Glezakos, Agathi. 2002. "Film Review: One Greek's reaction to My Big Fat Greek Wedding”. Reflections - Narratives of Professional Helping 8 (4): 69-72.

Hancock, Eleanor, and Craig Stockings, eds. 2013. Swastika over the Acropolis: Reinterpreting the Nazi Invasion of Greece in World War II. Leiden - Boston: Brill.

Hassapopoulou, Marina. 2007. “It's All Greek to Me: Misappropriations of Greekness in American Mass Media”. Journal of the Hellenic Diaspora: 59-80.

Hope, William. 2009. “Gabriele Salvatores' Marrakech Express and Mediterraneo: Capitalist Dystopias, the Marxist Sublime, Nascent Radicalism”. Italianist 29: 115-131. doi: 10.1179/026143409X409819.

Hudson, Simon, and J.R. Brent Ritchie. 2005. "Promoting Destinations via Film Tourism: An Empirical Identification of Supporting Marketing Initiatives". Journal of Travel Research 44 (4): 387-396. doi: 10.1177/0047287506286720.

Iancu, Anca-Luminita. 2017. "Guess Who's Coming to Dinner: Race, Ethnicity, and Women's Choices in Something New and My Big Fat Greek Wedding". East-West Cultural Passage 1: 50-72.

Kapiki, Soultana Tania. 2012. "Current and Future Trends in Tourism and Hospitality: The Case of Greece”. International Journal of Economic Practices and Theories 2 (1): 1-12.

Pallant, Chris. 2011. "Developments in Peplum Filmmaking: Disney's Hercules”. In Of Muscles and Men: Essays on the Sword and Sandal Film, edited by Michael G. Cornelius, 175-186. Jefferson: McFarland \& Company.

Rushing, Robert A. 2016. "Skin Flicks: Haptic Ideology in the Peplum Film". Cinema Journal 56 (1): 88-110. doi: 10.1353/cj.2016.0055.

Santarelli, Lidia. 2004. "Muted Violence: Italian War Crimes in Occupied Greece". Journal of Modern Italian Studies 9 (3): 280-299. doi: $10.1080 / 1354571042000254728$.

Schenk, Irmbert. 2006. "The Cinematic Support to National(istic) Mythology: The Italian Peplum 1910-1930". In Globalization, Cultural Identities and Media Representations, edited by Natascha Gentz and Stefan Kramer, 153168. Albany: State University of New York Press.

Tzanelli, Rodanthi, and Maximiliano E. Korstanje. 2016. “Tourism in the European Economic Crisis: Mediatised Worldmaking and New Tourist Imaginaries in Greece”. Tourist Studies 16 (3): 296-314. doi: 10.1177/1468797616648542.

Lingue Culture Mediazioni / Languages Cultures Mediation - 8 (2021) 1

https://www.ledonline.it/LCM-Journal/ - Online ISSN 2421-0293 - Print ISSN 2284-1881 
Tzanelli, Rodanthi. 2003. “'Casting' the Neohellenic 'Other': Tourism, the Culture Industry, and Contemporary Orientalism in 'Captain Corelli's Mandolin' (2001)”. Journal of Consumer Culture 3 (2): 217-244. doi: $10.1177 / 14695405030032004$.

Tzanelli, Rodanthi. 2004. “Europe' within and without Narratives of American Cultural Belonging in and through My Big Fat Greek Wedding (2002)”. Comparative American Studies - An International Journal 2 (1): 39-59. doi: 10.1177/1477570004041285.

Tzanelli, Rodanthi. 2017. "Staging Pilgrimage on Skopelos after Mamma Mia! (2008): Digital and Terrestrial Hospitality in Cinematic Tourism”. In Gazing at Death: Dark Tourism as an Emergent Horizon of Research, edited by Maximiliano E. Korstanje and Bintang Handayani, 131-260. New York: Nova Science Publishers.

Tzioumakis, Yannis, and Lydia Papadimitriou. 2015. "My Big Fat Life in Ruins: Marketing Greekness and the Contemporary US Independent Film”. In Film Marketing Into the Twenty-First Century, edited by Nolwenn Mingant, Cecilia Tirtaine, and Joël Augros, 36-46. London. Bloomsbury Publishing.

Tzioumakis, Yannis, and Lydia Papadimitriou. 2016. "Having Its Cake and Eating It Too: Contemporary American 'Indie' Cinema and My Big Fat Greek Wedding Reframed". In Indie Reframed: Women's Filmmaking and Contemporary American Independent Cinema. Traditions in American Cinema, edited by Linda Badley, 188-203. Edinburgh: Edinburg University Press.

Van Watson, William. 2005. "Escape from Patria(rchy), Return to the (M)other: The Italian Invasion of Greece in Mediterraneo and Captain Corelli's Mandolin”. Literature/Film Quarterly 33 (4): 313-321.

\section{Filmografia}

300. 2007. Directed by Zack Snyder. Stati Uniti d'America.

300: Rise of an Empire (300 - L'alba di un impero). 2014. Directed by Noam Murro.

Stati Uniti d'America.

Alexander. 2004. Directed by Oliver Stone. Stati Uniti d'America - Regno Unito Germania - Italia - Paesi Bassi.

Appartamento ad Atene. 2012. Diretto da Ruggero Dipaola. Italia - Germania.

Arrivano i titani. 1962. Diretto da Duccio Tessari. Italia - Francia.

Attenberg. 2010. Directed by Athina Rachel Tsangari. Grecia.

Before Midnight. 2013. Directed by Richard Linklater. Stati Uniti d'America.

Captain Corelli's Mandolin (Il mandolino del capitano Corelli). 2001. Directed by John Madden. Francia - Regno Unito - Stati Uniti d'America.

Che ne sarà di noi. 2004. Diretto da Giovanni Veronesi. Italia.

Lingue Culture Mediazioni / Languages Cultures Mediation - 8 (2021) 1

https://www.ledonline.it/LCM-Journal/ - Online ISSN 2421-0293 - Print ISSN 2284-1881 
Ercole al centro della Terra. 1961. Diretto da Mario Bava. Italia.

Ercole contro i tiranni di Babilonia. 1964. Diretto da Domenico Paolella. Italia.

Ercole e la regina di Lidia. 1950. Diretto da Pietro Francisci. Italia - Spagna - Francia.

Gli amori di Ercole. 1960. Diretto da Carlo Ludovico Bragaglia. Italia - Francia.

Hercules. 1997. Directed by John Musker and Ron Clements. Stati Uniti d'America.

Hercules: The Legendary Journeys (Hercules). 1995-1999. Directed by Christian Williams and Robert Tapert. Nuova Zelanda - Stati Uniti.

Il colosso di Rodi. 1961. Diretto da Sergio Leone. Italia - Spagna - Francia.

Immaturi - Il viaggio. 2012. Diretto da Paolo Genovese. Italia.

Kynodontas (Dogtooth). 2009. Directed by Yorgos Lanthimos. Grecia.

Le fatiche di Ercole. 1958. Diretto da Pietro Francisci. Italia - Spagna.

Mamma Mia!. 2008. Directed by Phyllida Lloyd. Stati Uniti d'America - Regno Unito - Germania.

Mediterraneo. 1991. Diretto da Gabriele Salvatores. Italia.

Miss Violence. 2013. Directed by Alexandros Avranas. Grecia.

My Big Fat Greek Wedding (Il mio grosso grasso matrimonio greco). 2002. Directed by Joel Zwick. Canada - Stati Uniti d'America - Grecia.

My Life in Ruins (Le mie grosse grasse vacanze greche). 2009. Directed by Donald Petrie. Stati Uniti d'America - Grecia - Spagna.

Suntan. 2016. Directed by Argyris Papadimitropoulos. Grecia.

Troy. 2004. Directed by Wolfgang Petersen. Stati Uniti d'America - Malta - Regno Unito.

Xena: Warrior Princess (Xena - Principessa Guerriera). 1995-2001. Directed by John Schulian and Robert Tapert. Nuova Zelanda - Stati Uniti.

Young Hercules. 1998-1999. Directed by Robert Tapert. Stati Uniti d'America Nuova Zelanda. 